\title{
Improvement of Hydrogen Peroxide Sensitivity by an Electrochemiluminescent Method with Luminol using Polyion Adsorption onto the Electrode
}

\author{
Yasuo YOSHIMI, ${ }^{\text {* }}$ Masayuki KAMADA, ${ }^{\text {a }}$ Yoshiyuki OHKAWARA, ${ }^{\mathrm{a}}$ \\ Koji HATTORI, ${ }^{\mathrm{b}}$ and Kiyotaka SAKAI ${ }^{\mathrm{b}}$
}

\author{
a Department of Applied Chemistry, Shibaura Institute of Technology (3-9-14 Shibaura, Minato-ku, Tokyo 108-8548, Ja- \\ pan)

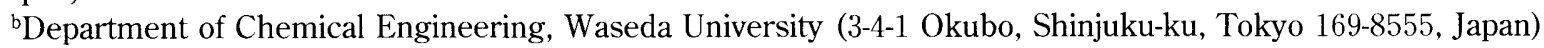

Received May 18, 2004 ; Accepted August 4, 2004

\begin{abstract}
Chemiluminescence $(\mathrm{CL})$ of luminol with hydrogen peroxide is useful for the highly sensitive determination of biochemicals by conjugation with oxidase. However, the method has stability problems because reactant-mixing conditions influence the results. However, electrochemiluminescence (ECL) of luminol can be performed on well-mixed solutions of luminol and hydrogen peroxide. ECL has not yet been applied to the quantitative analysis of biochemicals due to low quantum yields at physiological pH. In this work, we evaluated the effect of modifying the electrode with cationic polymer on the ECL intensity of luminol. Transparent indium-tin oxide (ITO) electrode was treated with 1 wt \% aqueous solution of polyethyleneimine ( $\mathrm{MW}: 70,000)$ buffered by $0.1 \mathrm{M}$ borate at $\mathrm{pH} 8.0$. ECL intensity at the ITO electrode was measured in a mixed solution of $1.0 \mathrm{mM}$ luminol and $10-30 \mu \mathrm{M}$ hydrogen peroxide buffered by phosphate or Tris- $\mathrm{HCl}(\mathrm{pH} \mathrm{7.4)}$. The electrode potential was applied in alternate pulses of $0.00 \mathrm{~V}$ versus $\mathrm{Ag} / \mathrm{AgCl}$ for $3 \mathrm{~s}$ and $1.00 \mathrm{~V}$ for $3 \mathrm{~s}$. The sensitivity of ECL to hydrogen peroxide concentration at the treated ITO increased remarkably. Results indicate that the cationic polymer adsorbed on the electrode enhances ECL by increasing pH in the vicinity of the electrode surface. However, the cationic polymer also enhanced inhibition of ECL by ascorbic acid, an anionic reducing reagent.
\end{abstract}

Key Words : Luminol, Electrochemiluminescence, Hydrogen Peroxide, Polyion, Sensitivity

\section{Introduction}

The reaction between luminol and hydrogen peroxide generates chemiluminescence in high quantum yield in the presence of catalyst (e.g., hemine, ferricyanide and peroxidase). This chemiluminescence is useful for the highly sensitive quantitative analysis of biochemicals (e.g., glucose, cholesterol and uric acid) that can be converted into hydrogen peroxide by an oxidase. ${ }^{1)}$ Additionally, luminol is inexpensive and possesses low toxicity. Nevertheless, luminol has not been used widely for the practical analysis of biochemicals because the chemiluminescence intensity depends on the mixing conditions of the hydrogen peroxide, luminol and catalyst solutions. Electrochemiluminescence $(\mathrm{ECL})$ is chemiluminescence generated by electrode instead of catalysis. ${ }^{2-7}$ ) When ECL is generated after complete mixing of luminol and hydrogen peroxide, ${ }^{6}$ ) it is an effective analytical method. The ECL is particularly applicable for micro total analytical systems ( $\mu$ TAS), in which the mixing process is hard to operate stably. However, the optimal $\mathrm{pH}$ for the ECL reaction of luminol is approximately 11, which is far from the optimal $\mathrm{pH}$ for oxidase reactions. ${ }^{5}$ ) The $\mathrm{pH}$ must be adjusted for application of the enzymatic and ECL reactions to the analysis of biochemicals. ${ }^{4}{ }^{4}$ ) However, the procedure requires mixing of buffer solutions, which is difficult to operate in $\mu$ TAS.
If the $\mathrm{pH}$ in the vicinity of the electrode surface can be increased, generation of the ECL is more intense at mild (physiological) $\mathrm{pH}$. We attempted to increase $\mathrm{pH}$ by modification of the electrode surface.

Indium-tin oxide (ITO) is optically transparent and electrochemically stable, and thus is an ideal electrode for ECL. The surface of ITO possesses a slight negative charge, allowing stable adsorption of a cationic polymer. Therefore, it is likely that an adsorbed cationic polymer will increase $\mathrm{pH}$ in the vicinity of the ITO electrode surface and enhance the ECL of luminol at mild $\mathrm{pH}$. In this work, the effect of cationic polymer adsorption on the sensitivity of ECL of luminol to hydrogen peroxide was estimated. Anionic polymer adsorbs onto the adsorbed cationic polymer to generate a stable and smooth layer. ${ }^{8-10)}$ We also observed the effect on the sensitivity of anionic polymer adsorption on the cationic polymer layer adsorbed directly on ITO.

\section{1 Materials}

\section{Experimental}

ITO sputtered glass plate (ITO layer thickness : 50 $\mathrm{nm}$ ) was purchased from Asahi Glass Co, Ltd. (Tokyo, Japan). Malachite green was purchased from Aldrich Chemical Co. (Milwaukee, USA). All other chemicals were purchased from Wako Pure Chemical Industries, 
Ltd. (Osaka, Japan).

2. 2 Modification of ITO by polyion adsorption

The ITO was prepared as described previously, ${ }^{11}$ and washed by a previously published procedure. ${ }^{12)}$ The ITO was soaked for $15 \mathrm{~min}$ in an aqueous solution of $1 \mathrm{wt} \%$ polyethyleneimine (PEI, MW : 70,000) buffered by $0.1 \mathrm{M}$ borate at $\mathrm{pH}$ 8.0. The treated ITO is designated PEI-ITO.

The PEI-ITO was soaked in an aqueous solution of 1 wt $\%$ polyacrylic acid (PAA, MW : 25,000) for $15 \mathrm{~min}$. The treated ITO is designated PAA-PEI-ITO. The change in surface charge of the treated ITO was confirmed by staining with an aqueous solution of $1 \mathrm{wi} \%$ anionic dye rosebengal or $1 \mathrm{wt} \%$ cationic malachite green containing $0.1 \mathrm{M} \mathrm{HCl}$. The surficial elemental compositions of unmodified ITO, PEI-ITO and PAA-PEI were determined by X-ray photoelectron spectroscopy (XPS : JPS-9010 TR, JEOL Ltd., Tokyo).

\section{3 ECL flow cell}

The ECL flow cell and system for ECL measurement procedures have been outlined previously. ${ }^{11}$ ) The ITO was fixed in the flow cell, ${ }^{11}$ in which a stainless tube was used as a counter electrode and an $\mathrm{Ag} / \mathrm{AgCl}$ electrode was used as a reference.

\section{4 ECL measurement}

Luminol $(0.1772 \mathrm{~g})$ was dissolved in $10 \mathrm{ml}$ of $0.1 \mathrm{M}$ sodium hydroxide, which was then neutralized by adding $0.1 \mathrm{M}$ hydrochloric acid and diluted by buffered solution (pH 7.4) of $0.1 \mathrm{M}$ phosphate or of $0.1 \mathrm{M}$ Tris- $\mathrm{HCl}$ to a total volume of $1,000 \mathrm{ml}$ (equivalent to a luminol concentration of $1.0 \mathrm{mM}$.) Hydrogen peroxide was added to a concentration of $0-30 \mu \mathrm{M}$.

The test solution was introduced into the ECL flow cell using a peristaltic pump (model PST-103 N, Asahi Techno Glass Ltd., Tokyo, Japan) at a flow rate of $3 \mathrm{ml} /$ min. The working electrode potential was applied in alternate pulses of $0.00 \mathrm{~V}$ versus the $\mathrm{Ag} / \mathrm{AgCl}$ reference electrode for $3 \mathrm{~s}$ and $1.00 \mathrm{~V}$ for $3 \mathrm{~s}$. Photons emitted from the ECL flow cell were detected by a photomultiplier tube (PMT : R 269, Hamamatsu Photonics Hamamatsu, Japan) connected to a digital ammeter (model AM-271 A, TOA Electric, Tokyo, Japan). The peak of PMT current intensity measured by the ammeter during the positive potential application was sampled as the ECL intensity. The relation between photocurrent intensity and hydrogen peroxide concentration was compared among the three ITO electrodes.

\section{1 Surface characterization}

\section{Results and Discussion}

The anionic rosebengal stained only PEI-ITO and the cationic malachite green stained only PAA-PEI-ITO, indicating that the ionic polymers PEI and PAA generate a positively and negatively charged layer, respectively, on the ITO surface. The surface elemental compositions of the unmodified and modified ITO as determined by XPS are shown in Table 1 . Treatment with PEI decreased the relative surface content of indium and tin, while dramatically increasing those of carbon and nitrogen. Furthermore, PAA treatment clearly decreased the contents of indium, tin and nitrogen. These results support the for-
Table 1 Surface elemental composition of the unmodified and modified ITO, determined by XPS.

\begin{tabular}{lrrrrr}
\hline \multirow{2}{*}{ ITO } & \multicolumn{5}{c}{ Surface elemental composition [\%] } \\
\cline { 2 - 6 } & \multicolumn{1}{c}{ In } & Sn & O & C & N \\
\hline Unmodified & 20.9 & 3.0 & 36.4 & 37.9 & 1.8 \\
PEI- & 8.3 & 1.3 & 27.9 & 59.0 & 3.5 \\
PAA-PEI- & 3.0 & 0.4 & 28.1 & 65.6 & 2.8 \\
\hline
\end{tabular}
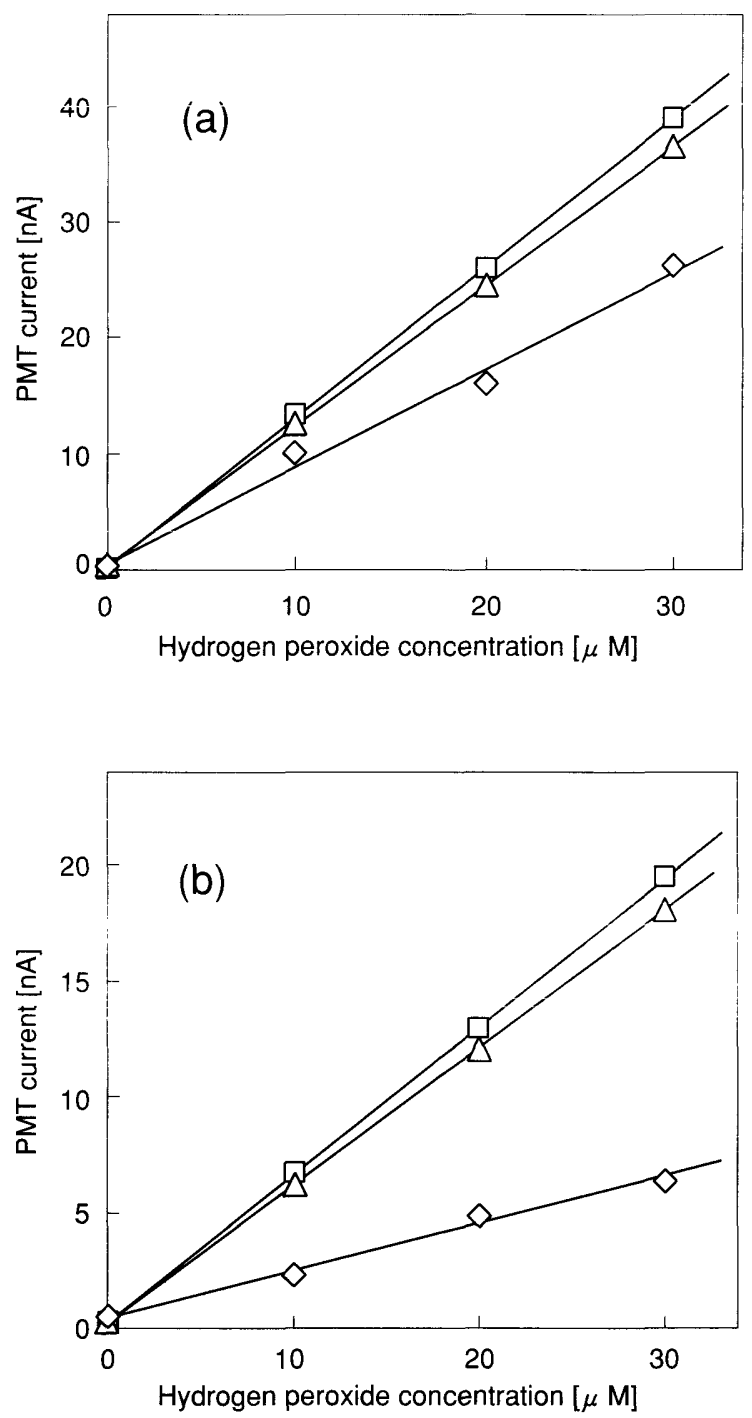

Fig. 1 Relation between PMT current intensity and hydrogen peroxide concentration buffered by (a) $0.1 \mathrm{M}$ Tris- $\mathrm{HCl}$ or (b) $0.1 \mathrm{M}$ phosphate at $\mathrm{pH}$ 7.4. Luminol concentration: 1.0 mM. Electrodes: Unmodified ITO (diamond), PEI-ITO (square), or PAA-PEI-ITO (triangle).

mation of a polyion adsorbing layer on the ITO. The thickness of the polymer-adsorption layer was less than $10 \mathrm{~nm}$, which is the maximum depth of detection by XPS, because the emitted electrons from indium and tin were detectable on the treated ITO.

\section{2 Effect on luminescence intensity}

The relation between PMT current at the electrodes and hydrogen peroxide concentration is shown in Fig. 1 $(n=10)$. The PMT current intensity was so reproducible that all error bars for the standard deviation of ECL in- 
Table 2 Relative slopes of PMT current as a first-order function of hydrogen peroxide concentration at the polyionadsorbed ITO electrode to that at the unmodified ITO $[-]$.

\begin{tabular}{lcc}
\hline \multirow{2}{*}{ Electrode } & \multicolumn{2}{c}{ Buffer Solution } \\
\cline { 2 - 3 } & $0.1 \mathrm{M}$ Tris- $\mathrm{HCl}$ & $0.1 \mathrm{M}$ Phosphate \\
\hline PEI-ITO & 1.5 & 3.2 \\
PAA-PEI-ITO & 1.4 & 3.0 \\
\hline
\end{tabular}

tensity data are concealed under the plot. A high degree of linearity existed between current intensity and hydrogen peroxide concentration in the range of $0-30 \mu \mathrm{M}$. The slope of the line is a suitable parameter to estimate sensitivity of ECL to hydrogen peroxide concentration. The relative slopes at the polyion-adsorbed ITO electrode to that at unmodified ITO are listed in Table 2.

Results demonstrate that ECL intensity to hydrogen peroxide concentration increased approximately 1.5 and 3 times by adsorption of PEI in the solution buffered by Tris- $\mathrm{HCl}$ and phosphate, respectively. PAA adsorption decreased the sensitivity only slightly, indicating that adsorption of cationic polymer PEI on the electrode is effective for increasing the sensitivity of ECL intensity to hydrogen peroxide. This result can be explained by an increase of $\mathrm{pH}$ in the vicinity of the electrode surface. It is unlikely that optically transparent PAA absorbed a photon emitted from luminol $(425 \mathrm{~nm})$. However, PAA adsorption on the PEI layer may decrease ECL intensity by decreasing $\mathrm{pH}$ in the vicinity of the electrode surface. This effect is likely to be minimal due to the thinness of the layer in this experiment. Additionally, direct grafting poly (methacrylic acid) onto the ITO electrode by the procedure described previously ${ }^{131}$ quenched the ECL completely, indicating that the acidity or alkalinity of the polymer directly adsorbed onto the ITO strongly affected ECL intensity.

The possibility that permeation of strongly buffering anions (especially phosphate ions) into the adsorbed PEI layer might reduce the effect of $\mathrm{PEI}$ on $\mathrm{pH}$ at the electrode surface and on ECL intensity prompted additional investigation. We expected that the anionic PAA on the PEI layer would block permeation of anions by electrostatic repulsion and would enhance the PEI effect on ECL intensity. However, the ECL was enhanced dramatically by PEI in phosphate buffered solution compared to Tris-HCl buffered solution in which the anion has weak buffering capacity. These results suggest that the enhancement of ECL by PEI is not due to only pH increasing. Some additional factors, such as an increase in the longevity of intermediate products (especially radicals or excited products), may be important. The reaction path and the rates of each step of the ECL at the polyionmodified electrode in several electrolytes are needed to understand the effect of polyion adsorption on the ECL.

The adsorption of cationic polymers on the working electrode remarkably enhanced ECL between hydrogen peroxide and luminol. This simple adsorption method is useful for determining hydrogen peroxide usage at mild $\mathrm{pH}$ and for the microanalysis of biochemicals that produce hydrogen peroxide upon exposure to oxidases. The
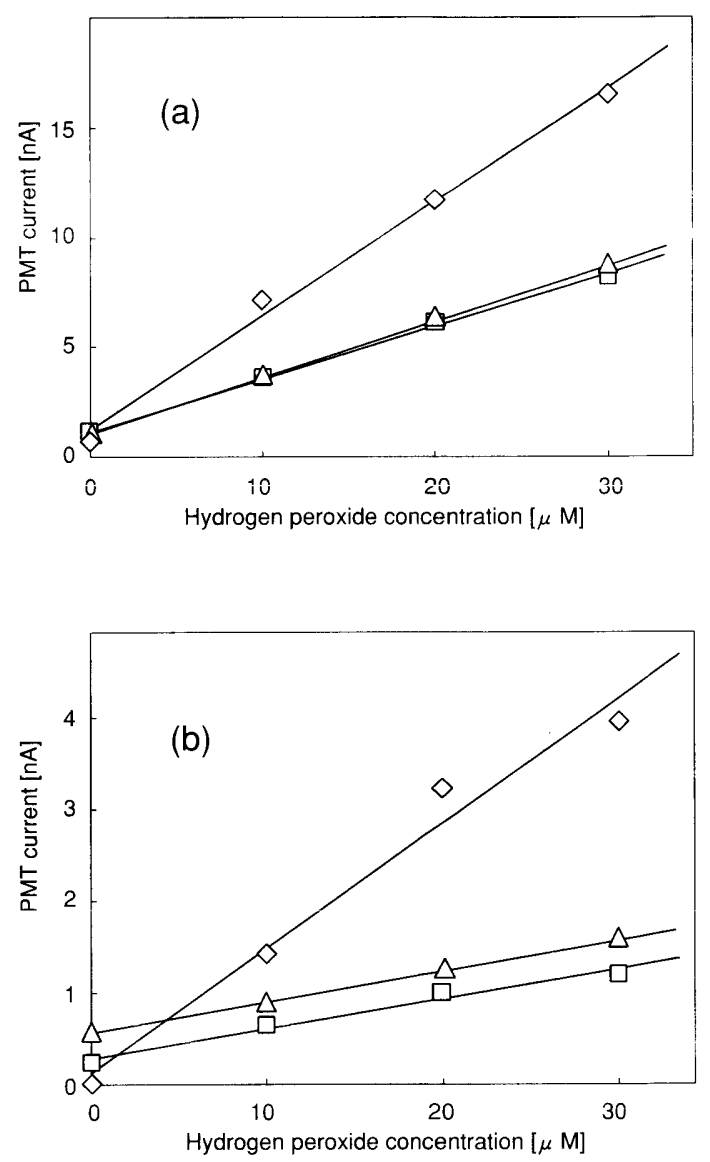

Fig. 2 Relation between PMT current intensity and hydrogen peroxide concentration in the presence of $100 \mu \mathrm{M}$ ascorbic acid. Buffer: (a) $0.1 \mathrm{M}$ Tris- $\mathrm{HCl}$ or (b) $0.1 \mathrm{M}$ phosphate at pH 7.4. Luminol concentration: $1.0 \mathrm{mM}$. Electrodes: Unmodified ITO (diamond), PEI-ITO (square), or PAA-PEI-ITO (triangle).

microanalysis system can be simplified because the enzymatic reaction and ECL can be operated at the same $\mathrm{pH}$. Modification of the electrode surface by a denser layer of cationic polymer may increase the sensitivity further.

\section{3 Effect of reductive anion}

A comparison of PMT current of three electrodes in the presence of ascorbic acid is shown in Fig. 2. The sensitivity of ECL intensity to hydrogen peroxide concentration is shown in Table 3. Results indicate that ECL intensity and its sensitivity to hydrogen peroxide at polymer-adsorbed ITO is decreased by ascorbic acid more dramatically compared to those at the unmodified ITO, due to the high affinity of anionic ascorbic acid on

Table 3 Relative decrease of the slope of PMT current as a first-order function of hydrogen peroxide concentration by adding $100 \mu \mathrm{M}$ ascorbic acid $[\%]$.

\begin{tabular}{lcc}
\hline \multirow{2}{*}{ Electrode } & \multicolumn{2}{c}{ Buffer Solution } \\
\cline { 2 - 3 } & Tris-HCl & Phosphate \\
\hline Unmodified ITO & 38 & 30 \\
PEI-ITO & 81 & 95 \\
PAA-PEI-ITO & 79 & 95 \\
\hline
\end{tabular}


cationic PEI. The results also indicate that the PAA layer on the PEI layer cannot prevent the effect of ascorbic acid on the PEI-modified ITO electrode by electrostatic repulsion against our attempt.

Body fluids (blood, urine, lymph fluid) are rich in anionic reducing chemicals (e.g., ascorbic acid and uric acid) that inhibit ECL of luminol at electrodes modified with cationic polymer. This property is a serious disadvantage for the practical application of electrode surface modification for highly sensitive quantification of biochemicals in body fluids. A method to prevent adsorption of reducing anions on the cationic layer is needed for practical application. Although anionic polymer adsorption on the cationic polymer layer was expected to be effective, results indicated that PAA adsorption was not effective for this purpose. Coating Nafion on an electrode dropwise is a widely applied method for blocking anionic reducing chemicals from electrodes. ${ }^{14,15)}$ However, the layer generated by this method usually has a thickness on the order of $\mu \mathrm{m}$. So, it is reasonable to assume that the anodic Nafion layer would inhibit ECL. Removal of the reducing agents by enzymatic pre-reactor ${ }^{16)}$ may enable analysis of biochemicals in body fluids using ECL at a PEI-modified ITO electrode.

\section{Conclusion}

ECL of luminol at the ITO electrode with adsorbed cationic polymer PEI is effective for highly sensitive determination of hydrogen peroxide at neutral $\mathrm{pH}$. This electrode modification is accomplished through a simple procedure that enables wide application of ECL for microanalysis of biochemicals by applying immobilized oxidases. However, the effect of PEI-adsorption on the ECL cannot be explained by a $\mathrm{pH}$ increase in the vicinity of the electrode alone. A study of the mechanism of ECLenhancement is needed to improve quantification of hydrogen peroxide by $\mathrm{ECL}$ at mild $\mathrm{pH}$.
Unfortunately, ECL via the PEI-modified electrode is inhibited by anionic reducing agents very seriously.

\section{References}

1) F. McCapra, Biosensors (Eds. A. P. F. Turner, I. Karube, and G.S. Wilson), Oxford University Press, Oxford, p. 617 (1987).

2) N. Harvey, J. Phys. Chem., 33, 1456 (1929).

3) T. Kuwana, J. Electroanal. Chem., 6, 164 (1963).

4) D. A. van Dyke, PhD Thesis, University of Illinois, Urbana, IL, USA (1986).

5) K. E. Haapakka and J. J. Kankare, Anal. Chim. Acta, 138, 263 (1982).

6) Y. Yoshimi and K. Sakai, Trans. Inst. Chem. Eng. Part C, 71, 279 (1993).

7) Y. Yoshimi, N. Himi, T. Kanamori, and K. Sakai, Biochemical Engineering for 2001, (Eds. S. Furusaki, I. Endo, and R. Matsumoto), Springer-Verlag, Tokyo, p. 629 (1992).

8) P. Ganguly, D. V. Paranjape, M. Sastry, S. K. Chaudhari, and K. R. Patil, Langmuir, 9, 487 (1993).

9) P. T. Hammond and G. M. Whitesides, Macromolecules, 28, 7569 (1995).

10) G. Decher, Science, 277, 1232 (1997).

11) Y. Yoshimi, H. Haramoto, T. Miyasaka, and K. Sakai, J. Chem. Eng. Jpn., 29, 851 (1996).

12) Y. Yoshimi, R. Ohdaira, C. Iiyama, and K. Sakai, Sensors and Actuators B., 73, 49 (2001).

13) K. Hattori, M. Hiwatari, C. Iiyama, Y. Yoshimi, F. Kohori, K. Sakai, and S. A. Piletsky, J. Membr. Sci., 233, 169 (2004).

14) J.-J. Xu, Z.-H. Yu, and H.-Y. Chen, Anal. Chim. Acta, 463, 239 (2002).

15) T. Matsumoto, M. Furusawa, H. Fujiwara, Y. Matsumoto, and N. Ito, Sensors and Actuators B: Chemical, 49, 68 (1998).

16) K. Hayashi, R. Kurita, T. Horiuchi, and O. Niwa, Biosensors \& Bioelectronics, 18, 1249 (2003). 